\title{
Ex-Situ Analysis and In-Situ Environmental TEM Studies of Manganite Perovskites for Catalytic Water Splitting
}

\author{
Christoph Luderer ${ }^{1}$, Stefanie Mildner ${ }^{1}$, Daniel Mierwaldt ${ }^{1}$, Julius Scholz ${ }^{1}$, Garlef Wartner ${ }^{1}$, Marcel \\ Risch $^{1}$ and Christian Jooss ${ }^{1}$ \\ 1. Institute of Materials Physics, University of Göttingen, Friedrich-Hund-Platz 1, 37077 Göttingen, \\ Germany.
}

Catalytic water splitting, i.e. the electrochemical decomposition of water into molecular hydrogen and oxygen allows for conversion and storage of solar or electrical energy in chemical energy and, thus, represents an important contribution to the development of renewable energy sources. The oxygen evolution half reaction $2 \mathrm{H}_{2} \mathrm{O}+4 \mathrm{~h} \rightarrow 4 \mathrm{H}^{+}+\mathrm{O}_{2}$ is the bottleneck of water-splitting since it requires the coupled transfer of 4 electron holes from the catalyst surface to the water molecule. This half reaction was comprehensively studied on perovskite catalysts [1], because they provide high chemical flexibility and the opportunity to selectively tune and adjust the electronic structure to the water oxidation potential by A- and B-site doping. Furthermore, electronic and electron-lattice correlations influence the electronic and crystal structure and may result in the formation of polaronic charge carriers, well known in particular for manganites. However, the impact of such correlation effects on catalytic activity is not yet understood.

Two manganite thin films were investigated. Firstly $\operatorname{Pr}_{0.65} \mathrm{Ca}_{0.35} \mathrm{MnO}_{3}$ (PCMO), which has strong octahedral tilting and a small bandwidth. Strong electron-electron and electron-phonon interactions lead to the formation of small polarons with hopping conductivity. In contrast to the strongly correlated PCMO, the second investigated manganite $\mathrm{La}_{0.67} \mathrm{Sr}_{0.33} \mathrm{MnO}_{3}$ (LSMO) shows weak correlation effects due to a larger bandwidth and metallic conductivity with large polaron charge carriers.

The comparative study of both contrasting manganites may contribute to a better understanding of the role of correlation effects and their impact on electrochemical processes at the manganite-water interface.

Preliminary ex-situ cyclovoltammetry and chronoamperometry measurements (at $\mathrm{pH}$ 7) show weak catalytic activity of PCMO accompanied by structural and chemical decomposition as revealed by post mortem scanning electron microscopy studies [2]. For LSMO, however, post-mortem analysis using XPS, XRD and AFM demonstrate high structural and morphological stability $[3,4]$ at higher catalytic activity than PCMO, which was further supported by extensive HRTEM analysis. To elucidate the chemical, structural and electronic processes at the atomic level underlying catalytic activity as well as degradation, in-situ transmission electron microscopy experiments were carried out in water vapor $\left(\mathrm{p}_{\mathrm{H} 2 \mathrm{O}}=3 \mu \mathrm{bar}\right)$.

First results at PCMO surfaces in contact with water vapor show that i) an electron beam induced potential can be used for driving an electrochemical reaction in the TEM, ii) a marker material in the environmental transmission electron microscopy (ETEM) can be used to visualize oxygen evolution reaction [5] and iii) oxygen evolution activity at PCMO surfaces involves significant changes in the electronic and atomic structure, which we interpret in terms of dynamic oxygen vacancy formation during catalytic activity [6,7]. The next step is to test the stability of LSMO in water vapor environment by ETEM. 


\section{References:}

[1] J Suntivich et al, Science 334 (2011), pp. 1383-1385.

[2] D Mierwaldt, Master's thesis, Institute of Materials Physics, University of Göttingen (2012).

[3] J Scholz et al, (2016) - in review.

[4] G Wartner, Master's thesis, Institute of Materials Physics, University of Göttingen (2016).

[5] S Mildner et al, J. Phys. Chem. C 119 (2015), pp. 5301-5310.

[6] S Raabe et al, Adv. Funct. Mater. 22 (2012), pp. 3378-3388.

[7] D Mierwaldt et al, Catalysts 4 (2014), pp. 129-145.

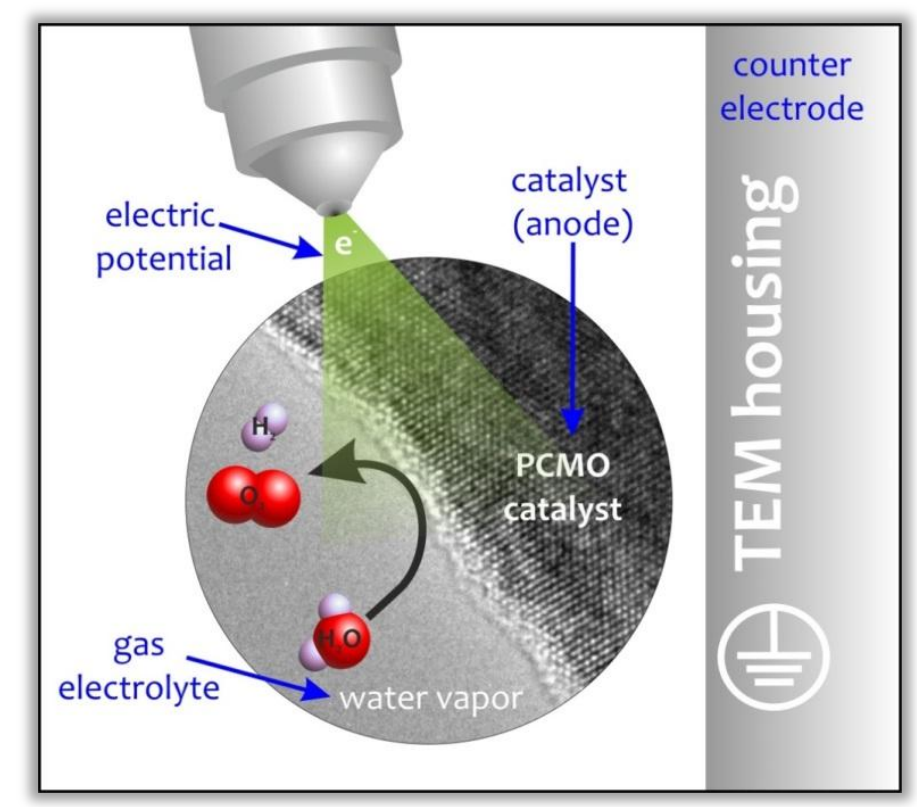

Figure 1. Scheme of the experimental setup in the ETEM. An electron beam induced potential is used for driving electrochemical reactions at the catalyst-water interface.
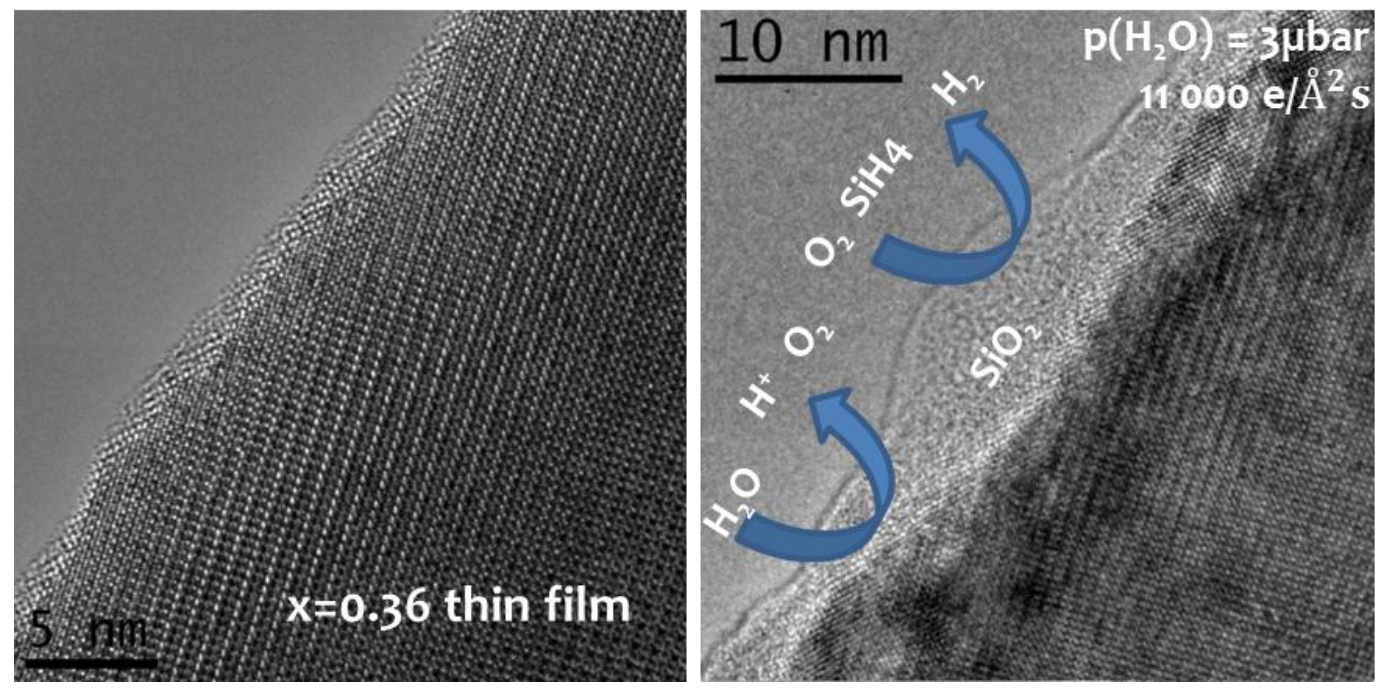

Figure 2. Crystalline edge of the PCMO film (a) in vacuum and (b) after $\mathrm{t} \sim 120 \mathrm{~s}$ in $\mathrm{H}_{2} \mathrm{O} / \mathrm{He} / \mathrm{SiH}_{4}$ gas $(\mathrm{p}=0.3 \mathrm{mbar})$ in an electron flux of $11000 \mathrm{e} /\left(\AA^{2} \mathrm{~s}\right)$. The growth of $\mathrm{SiO}_{2}$ in b) visualizes the electron beam induced oxygen evolution reaction [5], with permission from the American Chemical Society. 